\title{
Problématique de l'approvisionnement en eau potable dans la « mangroville» au sud de Douala, Cameroun
}

\author{
Safougne Djomekui Babette Linda, \\ Doctorante, département de Géographie, Université de Dschang, Cameroun \\ Aristide Yemmafouo, \\ $\mathrm{PhD}$, Maître de Conférences, Département de Géographie, \\ Université de Dschang, Cameroun \\ Dzalla Ngangue Guy Charly, \\ $\mathrm{PhD}$, Chargé de Cours, Université de Douala, Cameroun
}

URL:http://dx.doi.org/10.19044/esj.2020.v16n2p11

\section{Résumé}

Le problème d'approvisionement en eau potable dans les mangroves urbanisées semble occulté par les questions de dégradation de la couverture de mangrove bien plus médiatisés. Pourtant, ces nouveaux espaces urbains souffrent d'un déficit chronique d'eau potable accentué par l'insalubrité du milieu, l'abscence d'adduction d'eau et le fort taux de salinité de la nappe d'eau accessible aux habitants. L'étude a pour objectif de mettre en évidence le déficit en eau potable et ses implications sur la vie des habitants de la "mangroville» au Sud de Douala. Elle s'appuie sur des enquêtes par questionnaires auprès de 90 ménages, des entretiens avec les administrateurs locaux, des observations et des analyses physico-chimiques de trois échantillons d'eau prélevés sur le site. L'analyse des données révèle qu'en l'absence de connexion au réseau formel d'adduction d'eau potable, les habitants de mangroves urbanisées s'approvisionnent dans des puits et forages réalisés sans considérations des contraintes du milieu naturel. La conséquence est la prolifération des maladies hydriques, dont $62 \%$ de maladies de la peau et ulcères, $20 \%$ de gastro-entérites ou diarrhées, $8 \%$ de thyphoïde et $10 \%$ de paludisme. Au final, malgré les mesures prises pour apprivoiser l'eau en général, les habitants de «mangroville » n'ont pas réussi à assurer une qualité de l'eau potable capable de réduire le taux de prévalence des maladies hydriques dans leur cadre de vie. L'étude contribue à montrer que dans les villes du sud, l'occupation des sites non appropriés est un handicap majeur au développement urbain.

Mots-clés : Approvisionnement, Douala, eau potable, «mangroville », maladies hydriques, vulnérabilité 


\title{
Drinking Water Supply Issues in Mangrove Urbanized Area at the Southern Part of Douala, Cameroon
}

\author{
Safougne Djomekui Babette Linda, \\ Doctorante, département de Géographie, Université de Dschang, Cameroun \\ Aristide Yemmafouo, \\ $\mathrm{PhD}$, Maître de Conférences, Département de Géographie, \\ Université de Dschang, Cameroun \\ Dzalla Ngangue Guy Charly, \\ $\mathrm{PhD}$, Chargé de Cours, Université de Douala, Cameroun
}

\begin{abstract}
The problem of drinking water supply in urbanized mangrove area is overshadowed by other issues such as the degradation of the mangrove vegetation. Yet these new evolving urban areas suffer from a chronic lack of drinking water accentuated by an unsanitary environment, the absence of water supply network, as well as the high salinity of the water table accessible to its population. The study aims at demonstrating this chronic deficit and its implications on the livelihood of the "mangroville" in the southern part of Douala-Cameroon. The study is based on a questionnaire survey on 90 households, an interview with the local authorities, field observations and a physico-chemical analysis of three (03) water samples collected on site. Results indicate that, in the absence of a formal drinking water supply network, the residents of the mangrove urbanized area are supplied by wells and boreholes, hastily constructed without any consideration of environmental constraints. This leads to the proliferation of waterborne diseases, with $62 \%$ of skin diseases and ulcers identified in the surveyed sample, $20 \%$ of gastroenteritis or diarrhea, $8 \%$ of typhoid and 10\% of fever. Despite the measures taken to tame water, the inhabitants of these areas have failed to ensure the quality of drinking water, able to reduce the prevalence rate of waterborne diseases in their environment. The study contributes to show that in southern cities, the occupation of inappropriate sites is a major handicap to urban development.
\end{abstract}

Keywords: Douala, drinking water, "mangroville”, Supply, vulnerability, waterborne diseases 


\section{Introduction}

Le déficit d'approvisionnement en eau potable fait l'objet de plusieurs problématiques plus ou moins développées dans la littérature. Les hydrologues l'abordent par les facteurs naturels, notamment l'inégale répartition de l'eau à la surface du globe et la sécheresse (Bechler-Carmeau et al 2002); les techniciens évoquent les contraintes techniques comme le manque d'infrastructures de captage et de distribution de l'eau, les casses, la vétusté et la détérioration rapide des matériaux (Tegofack 2000 ; Ngali 2000 ; Katte 2006). Les socio-économistes quant à eux postulent que la pauvreté ne permet pas aux agences de distribution de l'eau d'étendre le réseau par rapport à l'explosion urbaine. En outre, le coût élevé des abonnements disqualifie les ménages à faible revenu qui constituent pourtant plus de $80 \%$ de la population des «mangrovilles». S'inscrivant dans cette approche, nous étudions le déficit d'approvisionnement en eau dans les mangroves urbanisées comme facteur aggravant de production des inégalités en milieu urbain. D'un autre côté, la pollution diffuse des sources potentielles d'approvisionnement en eau par le rejet dans l'eau des déchets d'origine anthropique accentué par l'étalement urbain anarchique, contribue à dégrader la qualité de l'eau (Anova Evrade et al. 2016).

La «mangroville» est le résultat de la forme d'urbanisation développée dans les mangroves (Dzalla 2012). C'est un espace urbain mis en place au détriment de la mangrove et caractérisé par la proximité des actions de l'eau marine, et notamment les effets de la présence alternée de l'eau (marée haute versus marée basse) et les effets de la salinité élevée, des formes d'aménagement précaires (divers pilotis) et le déficit chronique d'accès aux services urbains de base parmi lesquels l'eau potable. Dans ces espaces, la disponibilité de l'eau n'est pas toujours conforme à l'usage souhaité. Mais puisqu'il s'agit d'espace non destiné à l'urbanisation, les habitants se limitent à leurs propres moyens pour accéder aux services urbains et notamment à l'eau potable. Entre puits sommairement aménagés et forages non conventionnés, la quête de l'eau potable est une gageure particulièrement accentuée par les contraintes du milieu. L'étude a pour objectif de mettre en évidence ce déficit chronique et ses implications sur la vie des habitants et notamment les maladies hydriques. Elle postule que malgré les mesures prises pour apprivoiser l'eau en général, les habitants de «mangroville » n'ont pas réussi à assurer une qualité de l'eau potable capable de réduire le taux de prévalence des maladies hydriques dans leur milieu. Après une démonstration de l'urbanisation par dépit des mangroves (I), l'analyse des formes d'approvisionnement en eau potable (II) permet de mesurer le déficit et d'anticiper sur les vulnérabilités et surtout les maladies hydriques auxquelles les habitants sont exposés (III). 


\section{L'approche de terrain et la «mangroville » au sud de Douala I.1- L'approche de terrain}

La recension bibliographique a permis d'améliorer notre connaissance du milieu de mangrove, de confirmer l'originalité de cette étude, mais aussi de faire le tour des problématiques liées à l'approvisionnement en eau potable comme les questions de disponibilité en eau, d'accessibilité et de gouvernance. L'enquête de terrain s'est déroulée entre avril 2015 et juillet 2016 au sud de Douala (fig.1). Les observations et les entretiens ont concerné l'ensemble de la zone tandis que la zone de Nsong Ngongang-Mboh Nkhon (Cité Berge, Boko), la plus représentative pour la problématique, a été retenue pour l'administration du questionnaire. L'enquête par questionnaire a justement concerné 90 ménages choisis selon un échantillonnage aléatoire systématique à pas de 5 ménages. Le choix s'est fait le long des ruelles et pistes desservant les ménages jusqu'au front des mangroves fraichement colonisées. Les observations se sont focalisées sur la description des paysages et l'analyse des comportements des ménages face aux problèmes d'eau. L'observation participante a d'ailleurs été utilisée dans le cas de l'appréciation du goût l'eau par la consommation. Nous avons résidé dans trois ménages de nos contacts pendant une semaine chacune (saison pluie et saison sèche) pour mieux vivre la situation du manque d'eau et les stratégies développées.

Un prélèvement des échantillons d'eaux des forages les plus fréquentés a été réalisé en utilisant le matériel préparé et conditionné pour l'occasion aux fins d'analyse pour mettre en évidence les paramètres physicochimiques et bactériologiques permettant de conclure de manière objective sur la qualité de l'eau consommée. L'enquête par questionnaire auprès des ménages couplée à la recension des informations contenus dans les registres des centres de santé du quartier a contribué à mettre la lumière sur la forte prévalence des maladies hydriques. D'un autre côté, les données et informations recueillies par le biais des services de la commune de douala 3ème, de la recherche bibliographique et de l'exploitation des images satellitaires nous ont permis d'apprécier l'évolution de l'anthropisation de l'espace à mangrove du Sud de Douala en général et de la zone d'étude en particulier.

\section{2- La «mangroville » dans l'urbanisation au sud de Douala}

L'accroissement des surfaces bâties est si rapide qu'elle remet en question l'hostilité du site de la ville de Douala, c'est-à-dire un site de plaine s'étendant sur des espaces non propices à l'habitat humain comme les mangroves. Le flou juridique en matière de propriété foncière a favorisé l'occupation de ces espaces amphibies par une couche de la population en manque de terre pour bâtir leur logement. Motivés par l'acquisition de terrain à moindre coût, et cherchant à résoudre les problèmes causés par la pauvreté, les «débrouillards» investissent donc un milieu aux caractéristiques physiques 
redoutables pour l'habitat humain. L'occupation des zones à mangrove commence timidement entre 1985 et 1990, années marquées par le début de la crise économique. Pendant la décennie 1990, il est noté une légère augmentation de la population. A partir de 2000, l'accroissement devient exponentiel avec un maximum entre 2006 et 2010 (fig.1).

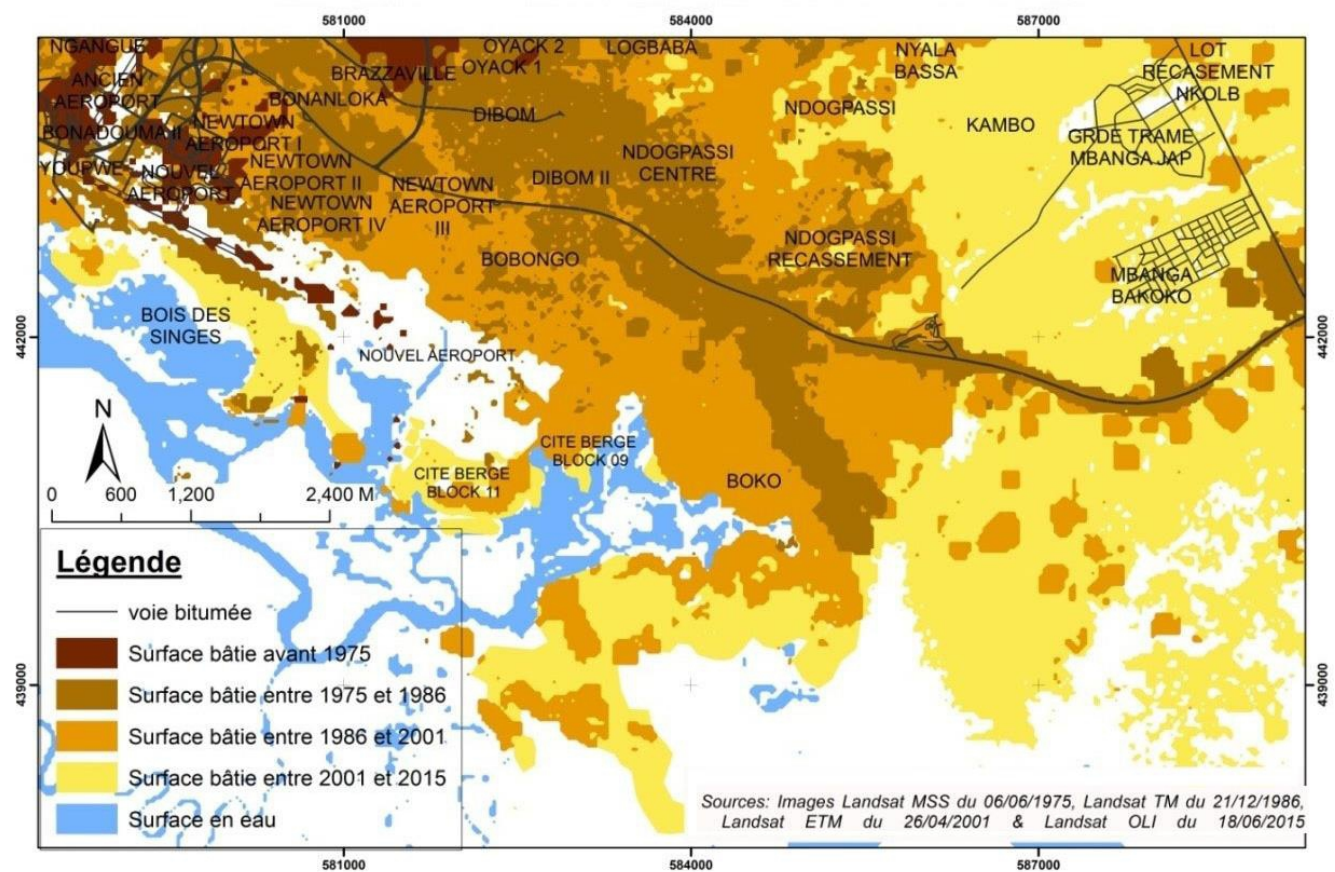

Figure 1 : Evolution des taches urbaines au Sud de Douala

Les premiers occupants de Nsong Ngongang-Mboh Nkhon furent prioritairement les pêcheurs et les exploitants de sables. Ils construisaient leurs maisons en matériaux provisoires et la plupart doutaient de la possibilité d'une implantation humaine «définitive » en raison des conditions naturelles très hostiles. Cependant, la pauvreté et la croissance démographique dans la ville de Douala a progressivement contraint à des installations définitives qui s'intensifient jusqu'à nos jours (fig. 2, fig.3). L'affermissement de ces installations donne naissance à des constructions en matériaux « définitifs » de plus en plus dominantes dans les zones exondées. La répartition des occupants par dates d'implantation apporte plus d'amples informations sur les tendances de la consommation spatiale (fig.3). Depuis les années 2000, le rythme d'occupation est assez soutenu. 
a. 2001

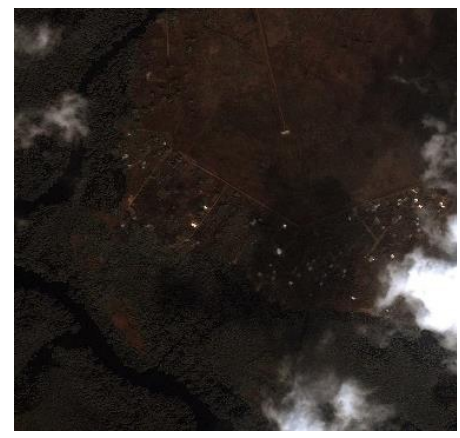

b. $\mathbf{2 0 0 7}$



c. $\mathbf{2 0 1 5}$

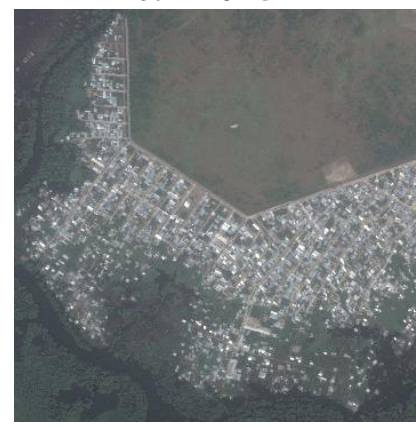

Figure 2: Images satellitaires de Nsong Ngongang captées à différentes périodes sur Google Earth

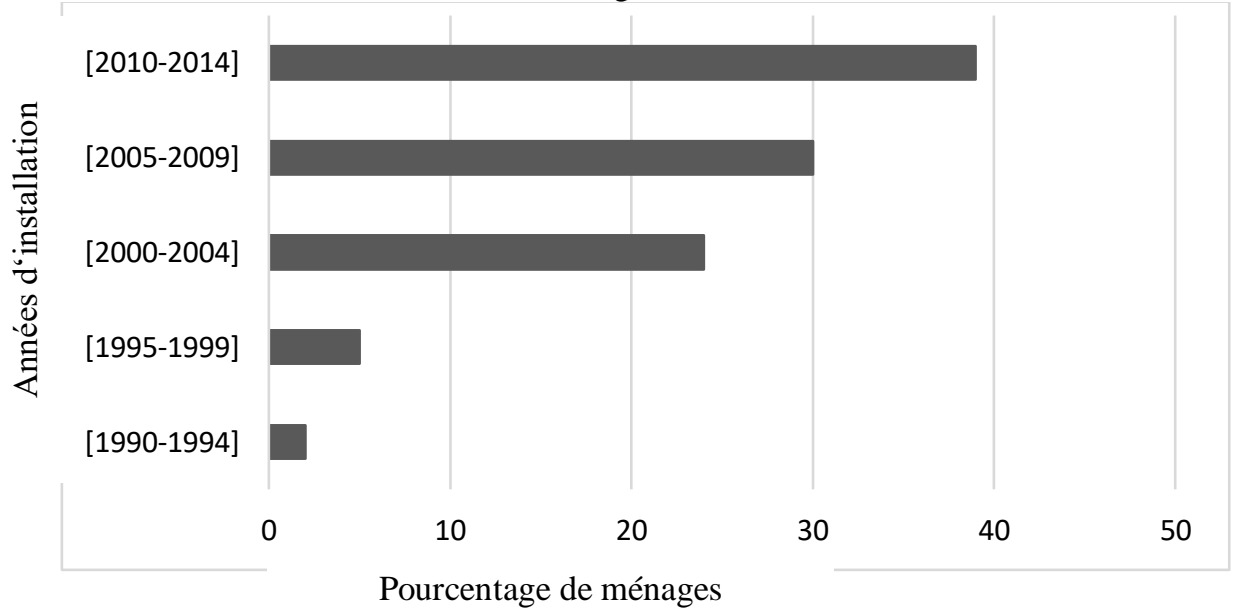

Figure 3 : Répartition des occupants de la mangrove de Nsong Ngogang-Mboh Nkon d'après les dates d'occupation, Source : Enquêtes de terrain, avril 2015

La « mangroville » de Nsong Ngogang-Mboh Nkon est à première vue comparable aux quartiers populeux des zones marécageuses situées dans la ville de Douala, mais il n'en est rien. En effet, même les populations venues des quartiers marécageux qui s'y sont installées ont pris du temps pour s'adapter aux contraintes physiques (instabilité du sol, mouvements de la marée et des vagues, etc.) qui rythment quasiment toutes les activités humaines. L'aménagement de ces espaces pour bâtir suppose le remblai en vue de défier la hauteur des eaux et de solidifier le sol (fig.4). 


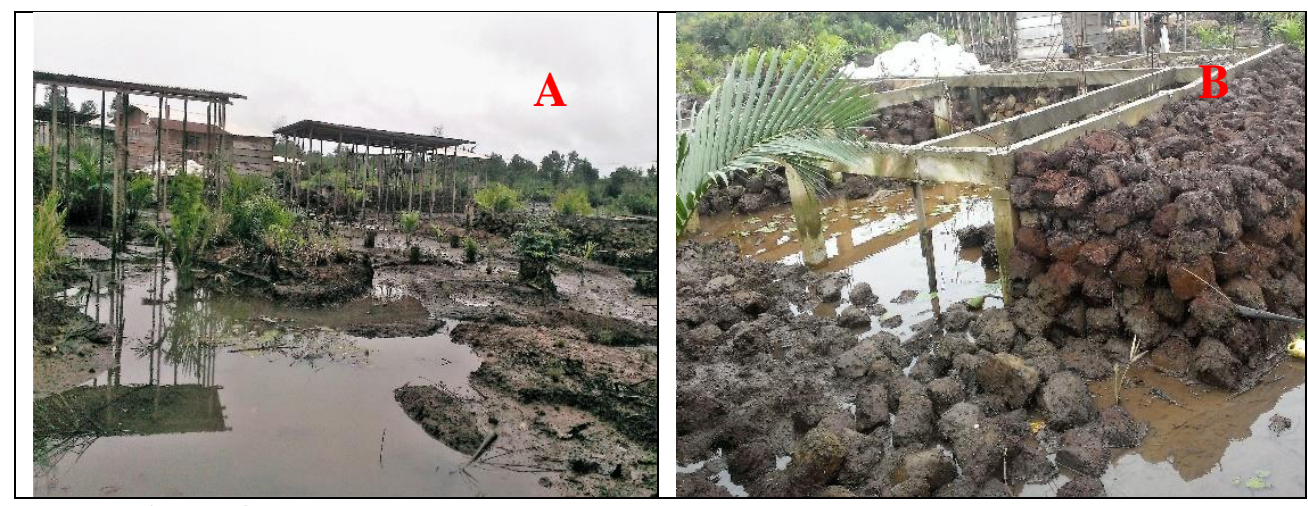

Figure 4 : Initiatives de construction sur le sol instable de Nsong Ngongang,

(Clichés, Safougne, mai 2015)

Cette figure illustre les aspects de l'environnement des «mangrovilles » et les initiatives d'aménagement entreprises par les habitants. A gauche (A), des restes de mangrove accueillant des pilotis sur un sol vaseux. Il est impossible d'envisager de creuser un puits ou un forage. A droite (B), pour palier à l'instabilité du sol, les plus fortunés font des remblaiements à base de morceaux de vase consolidée par les radicelles de pandanus. La structure et la résistance du sol sont étroitement liés à la dynamique des marées qui maintient le sol saturé et favorise la stagnation de l'eau en surface. La conséquence de cette implantation est l'enclavement qui met les résidents en marge de l'accès aux services de bases qu'offrent la ville et particulièrement l'eau potable. Cette ressource pourtant omniprésente devient paradoxalement une denrée bien rare. On assiste donc à une reproduction des modes précaires d'approvisionnement en eau, cette fois avec encore plus de risques.

\section{Des formes d'approvisionnement en eau caractéristique des quartiers précaires et amplifiées par les particularités du milieu}

\section{II.1- Des modes d'approvisionnement dominés par les puits sommaires}

Les ménages qui s'installent dans la «mangroville» sont pour la plupart des citadins venus d'anciens quartiers populeux de la ville de Douala. Ils sont motivés par le désir profond de sortir de la location pour devenir propriétaires d'une maison individuelle «à tous les prix» en fonction des pressions socioculturelles subies. L'occupation de la mangrove s'accompagne d'un transfert des pratiques d'approvisionnement en eau des quartiers précaires, à la différence qu'ici s'ajoute la contrainte du milieu de mangrove. Il existe prioritairement trois sources d'approvisionnement en eau à usage divers : le puits, le forage et l'eau de pluie. Les habitants ont pris l'habitude de faire des associations (une ou plusieurs sources) en fonction de l'usage, mais quel que soit le choix d'association, l'eau provenant des puits revient systématiquement. D'après nos enquêtes par questionnaires, $54,3 \%$ des 
ménages associent l'eau des puits et des forages pour tous leurs besoins ; $31,4 \%$ ajoutent à ces dernières l'eau de pluie ; $10 \%$ associent à ces trois dernières, l'eau minérale embouteillée ; et enfin, $3 \%$ seulement associent eau de forages, de puits et eau minérale (fig.5).

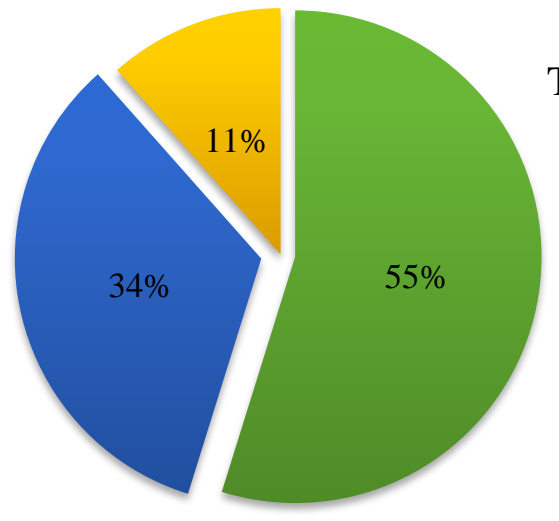

Type d'association

Puits, forage

Puits, forage, eau de pluie

$\square$ Puits, forage, eau minérale (embouteillée)

Figure 5: Répartition des sources d'approvisionnement en eau dans les ménages.

Source : Enquêtes de terrain, mai 2015

Le puits est l'infrastructure hydrique la plus présente dans les ménages dès que son aménagement est possible (fig.6). Les habitants sont convaincus que la terre filtre naturellement l'eau et justifient ainsi l'usage généralisé du puits comme «acceptable » en matière d'accès à l'eau. C'est justement les motifs de manque d'argent, de facilité d'accès et de mimétisme (habitude communautaire) qui ont concouru à la construction de cette perception de filtrage naturel de l'eau du sol.

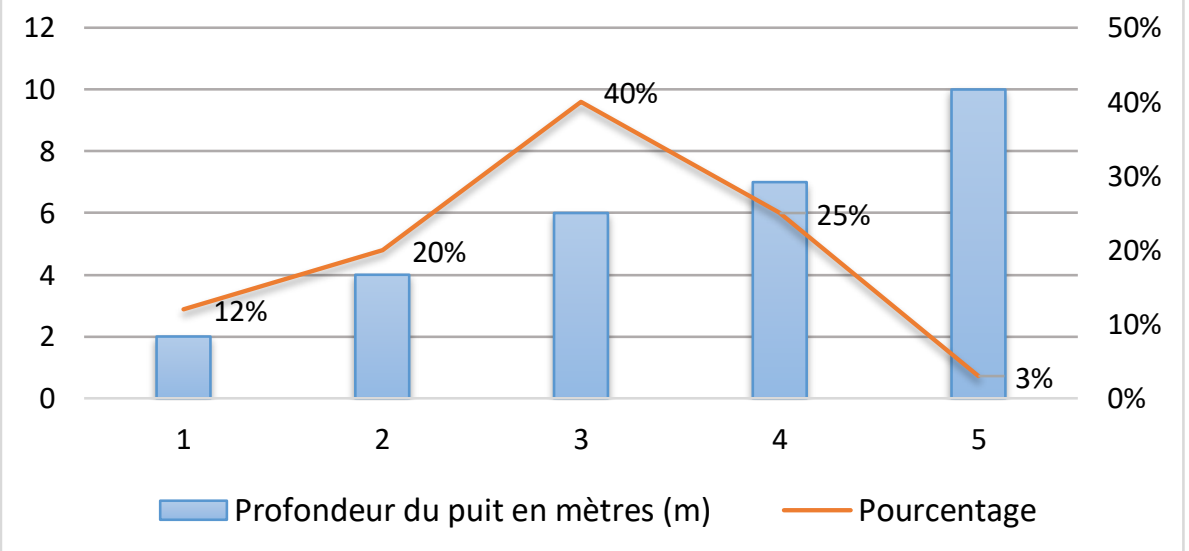

Figure 6 : Répartition de la profondeur des puits (en mètres) dans les ménages. Source : Enquêtes de terrain, mai 2015 
Lorsque les ménages parlent de puits, il s'agit d'installations sommaires (fig.7) de profondeurs variant entre 0 et $6 \mathrm{~m}$ maximum, donc constamment battues par la marée et les débris charriés par les courants de marées ou les inondations. En matière d'entretien, les meilleurs puits sont protégés par des buses (fig.7). Les buses limitent la suffosion ou le sapement des rebords et empêchent, soit les débris endogènes de polluer l'eau, soit carrément la fermeture du puits. Dans la plupart des cas, ceux qui n'ayants pas de puits contribuent à l'entretien du puits dans lequel ils se ravitaillent, cette contribution peut être matérielle (don de seau, corde, eau de javel, etc.) ou financière (500 à $1000 \mathrm{fcfa} / \mathrm{mo}$ is pour l'achat des désinfectants).

Les forages privés se retrouvent chez les plus nantis. Ils atteignent des profondeurs de 70m et ne sont pas exempts de défauts : la qualité de l'eau n'est pas optimale, et il y a aussi un risque de colmatage du forage lié à un sapement en profondeur en rapport avec la pression d'eau. Selon Paul, propriétaire de forage, "le coup d'implantation d'un forage avoisine 2.000.000 de FCFA et on n'est pas sûr de l'avoir toute la vie » (enquêtes de terrain, 2015).
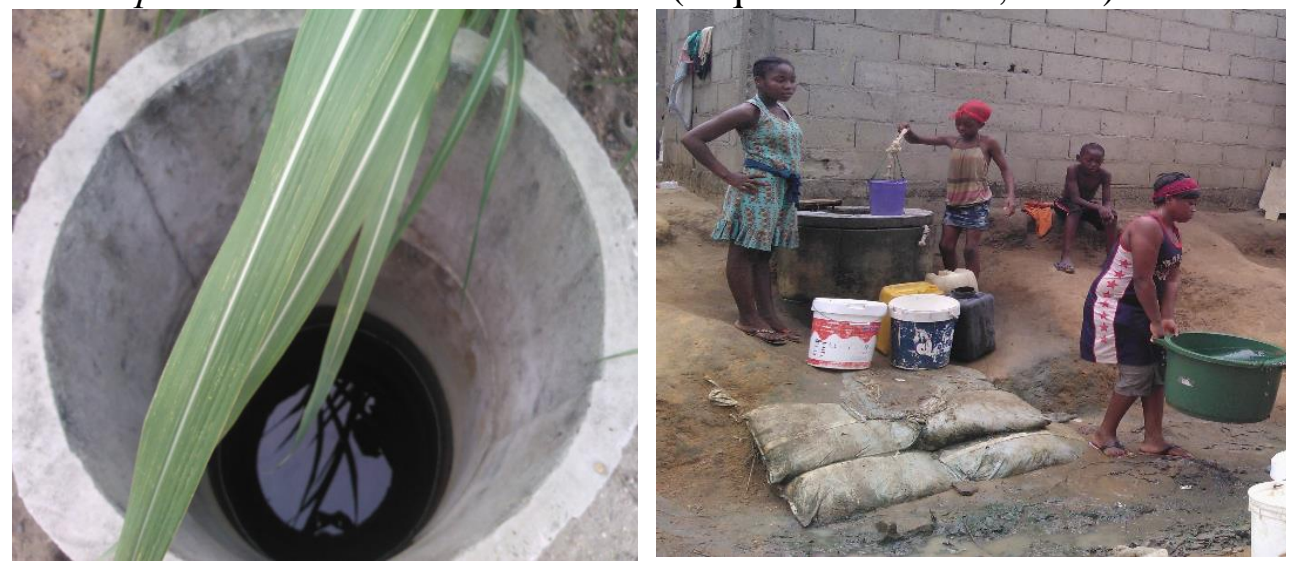

Figure 7 : Puits protégé par une buse (l'eau remonte presqu'à fleur de surface).

Clichés, Safougne, mai 2015

\section{II.2- Des traitements différenciés de l'eau potable en fonction du mode d'approvisionnement et des perceptions}

La situation de l'environnement à Nsong Ngogang montre en permanence des eaux stagnantes polluées par les déchets domestiques de tout genre (déchets fécaux des animaux et des humains). $85 \%$ des ménages pratiquent l'élevage porcin et les déchets sont directement déversés dans l'eau. Dans ce contexte, le traitement de l'eau, a un intérêt psychologique certain (fig.8). A la question de savoir: "traitez-vous votre eau de boisson à domicile? », $75 \%$ de ménages ont répondu systématiquement par l'affirmative. Seulement $25 \%$ consomment directement l'eau de forage. Ce résultat montre que les habitants sont conscients que l'eau qu'ils recueillent pourrait être impropre à la consommation. 


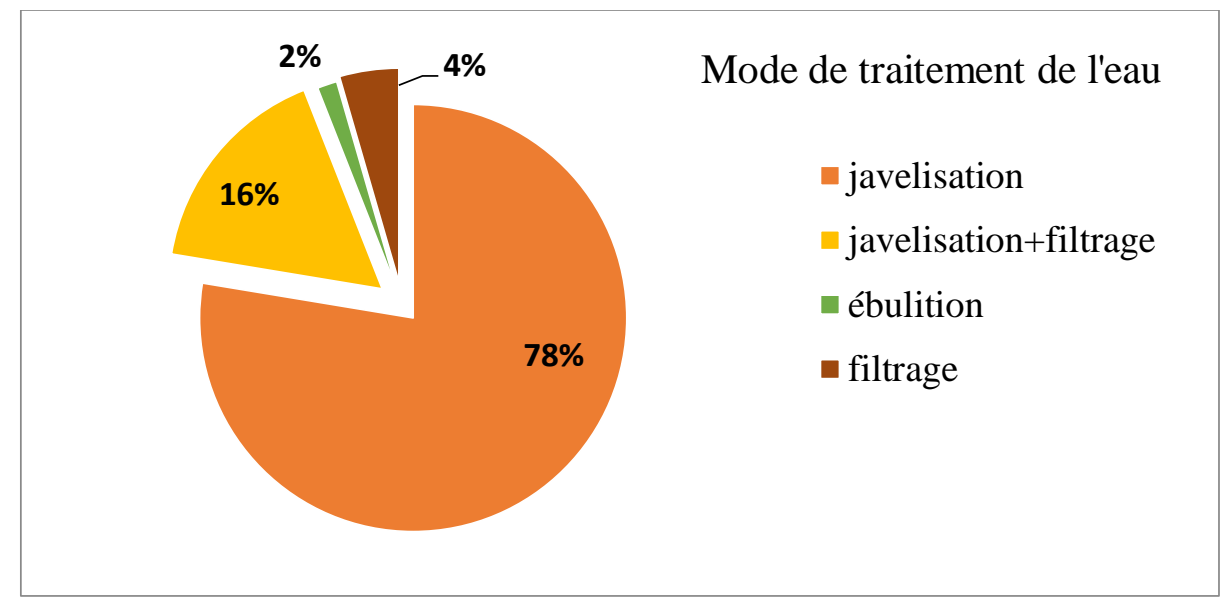

Figure 8: Répartition du mode de traitement de l'eau de boisson (forage) appliqué dans les ménages. Source : Enquêtes de terrain, mai 2015

Cette figure montre que le choix du mode de traitement de l'eau de forage destiné à la boisson varie en fonction des ménages, mais la pratique la plus répandue est la javellisation (78\%) à raison de 03 goutes de javel pour 10 litres d'eau. D'autre plus soucieux, associent à la javellisation, la filtration (16\%). Une minorité par contre se contente, soit de porter à ébullition uniquement (2\%), soit de filtrer uniquement (4\%). L'eau de puits bien que n'étant pas destiné à la boisson est aussi systématiquement traitée. Le désinfectant de référence ici est l'eau de javel qui est directement introduit dans le puits à raison d'un litre de javel dans le puits une fois par trimestre. A côté de ce produit, d'autres y associe le charbon (30\%), et dans certains cas le sel (15\%). L'habitant Jean, nous explique que pour l'entretien de son puits de 04 mètres de profondeur, il utilise deux sachets de javel en poudre et un demisac de sel, soit $9 \mathrm{~kg}$ pour «tuer» les microbes pendant 3 à 4 mois. Cette situation a conforté notre volonté d'en savoir plus sur la qualité de l'eau dans cette zone surtout en matière d'eau de boisson; ceci d'autant plus que la donnée qualité de l'eau a des enjeux qui dépassent les limites de notre zone d'étude.

\section{Construction des vulnérabilités et expansion des maladies hydriques III.1- Des cadres de vie inapproprié à la bonne conservation de l'eau}

Les risques de pollution et de contamination des eaux sont accentués par l'absence de latrines aménagées de façon convenable dans la plupart des ménages $(90 \%)$. Même lorsque des efforts d'aménagement sont fournis, la distance de ces latrines de fortune par rapport au point d'eau est dans la plupart des cas de l'ordre de moins 5 mètres (fig.9). 

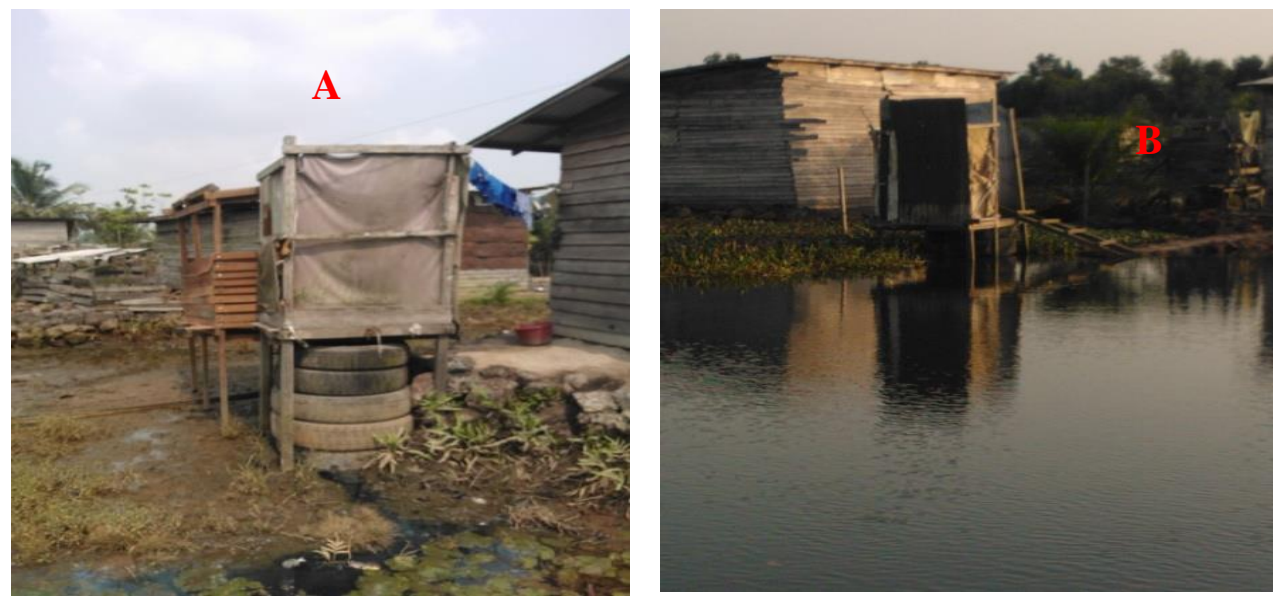

Figure 9 : Latrines sur pilotis à quelques mètres des puits. Cliché, Safougne, juillet 2015

La première image $(\mathrm{A})$ donne un aperçu des latrines aménagé après le retrait des eaux. La précarité de l'aménagement est marquée par de vielles roues de voiture empilées et un tissu pour se mettre à l'abri des regards. Pendant cette phase de basses eaux, les déchets fécaux s'acculent entre les roues superposées. La deuxième image (B) présente la même latrine pendant l'inondation. A ce moment l'eau joue le rôle de vidange naturelle en répandant çà et là les excréments humains. C'est ce qui explique la forte teneur en E.coli dans l'eau de boisson. À quelques mètres de ces latrines sont installés les puisards ou les puits traditionnels non aménagés et dans certains cas des forages. À tout moment de l'année, la circulation de l'eau peut entraîner une contamination par les microbes provenant des latrines. En saison des pluies et en cas d'inondation, les puits, mais aussi les mares et l'eau courante sont de véritables collecteurs d'eaux insalubres. Par ailleurs, l'absence de service de collecte des ordures ménagères contribue à la pollution de l'eau. En effet, les déchets de toutes natures provenant des activités des habitants y compris en amont sont tout simplement déversés dans l'eau et stagnent dès que la pente s'annule (fig.10). 


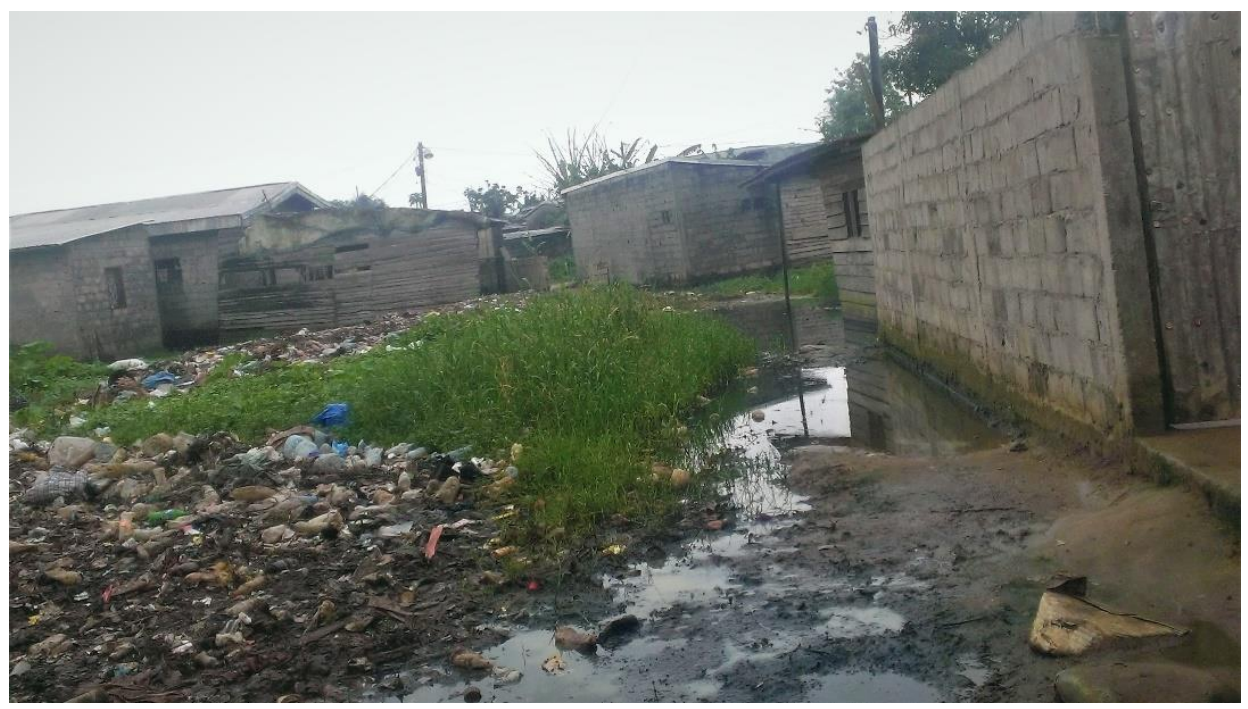

Figure 10 : Décharge d'ordures ménagères dans l'eau à proximité des habitations. Cliché, Safougne, juillet 2015

\section{III.2- Une qualité de l'eau potable médiocre ignorée par les habitants}

Lorsqu'ils évaluent la qualité de l'eau de boisson, les résidents se fient principalement à leurs sens (fig.11), c'est-à-dire que chaque ménage boit de l'eau qu'il estime être potable en fonction de ses critères ou de la perception de la majorité.



Raisons pour lesquelles les usagers trouvent l'eau potable

Figure 11 : Répartition des critères de potabilité tels que perçus par les habitants.

Source : Enquêtes de terrain, 2016

Pour pallier à cette évaluation pour le moins subjective de la potabilité de l'eau, des analyses physicochimiques et bactériologiques ont été réalisées et les résultats obtenus ont été enregistrés dans le tableau 1. 
Tableau 1 : Caractères physiques et bactériologiques observés dans les échantillons d'eaux prélevés dans trois forages de Nsong Ngongang-Mboh Nkhon

\begin{tabular}{|c|c|c|c|c|}
\hline \multicolumn{2}{|c|}{$\begin{array}{l}\text { prélevée } \\
\text { Paramètres } \\
\text { Analysés }\end{array}$} & Forage 1 & Forage 2 & Forage 3 \\
\hline \multicolumn{2}{|c|}{ Heure de prélèvement (matin) } & 08h30' & 08h50' & 09h1' \\
\hline $\begin{array}{l}\text { Caractères } \\
\text { physiques }\end{array}$ & $\begin{array}{l}\text { Normes OMS } \\
2010\end{array}$ & \multicolumn{3}{|c|}{ Paramètres observés } \\
\hline Apparence & Claire & Claire & Claire & Claire \\
\hline $\mathrm{PH}$ & $6,5-9$ & 5,0 & 5,4 & 6 \\
\hline $\begin{array}{l}\text { Conductivité } \\
\text { électrique }(\mu \mathrm{S} / \mathrm{cm})\end{array}$ & $\begin{array}{l}400- \\
1250 \mu \mathrm{S} / \mathrm{cm}\end{array}$ & 29,2 & 15,6 & 56,4 \\
\hline Odeur & Nulle & Nulle & Nulle & Nulle \\
\hline Gout & Nulle & $\begin{array}{l}\text { Relativement } \\
\text { désagréable }\end{array}$ & $\begin{array}{l}\text { Relativement } \\
\text { désagréable }\end{array}$ & $\begin{array}{l}\text { Relativement } \\
\text { désagréable }\end{array}$ \\
\hline Larves de parasites & Nulle & Nulle & Nulle & Nulle \\
\hline $\begin{array}{l}\text { Paramètres } \\
\text { bactériologiques }\end{array}$ & $\begin{array}{l}\text { Normes OMS } \\
2010\end{array}$ & \multicolumn{3}{|c|}{ Paramètres observés } \\
\hline Escherichia coli & $0 / 100 \mathrm{ml}$ & $100 / 100 \mathrm{ml}$ & $23 / 100 \mathrm{ml}$ & $23 / 100 \mathrm{ml}$ \\
\hline $\begin{array}{l}\text { Entérocoques } \\
\text { intestinaux }\end{array}$ & $0 / 100 \mathrm{ml}$ & $25 / 100 \mathrm{ml}$ & $84 / 100 \mathrm{ml}$ & $05 / 100 \mathrm{ml}$ \\
\hline
\end{tabular}

Source : analyses de laboratoire, centre pasteur du Cameroun, 2016

Les résultats ci-dessus montrent que l'eau de boisson en laquelle les habitants ont une grande confiance est fortement polluée et a une tendance à l'acidité. Les paramètres physiques révèlent une eau dont le P.H varie sensiblement de l'aval (forage1) vers l'amont (forage3). L'aval ici est caractérisé par la proximité des eaux de mangrove. La tendance générale est à l'acidité, soit respectivement : 5,$0 ; 5,1 ; 6,1 ;$ ce qui est hors normes. D'après les données de l'OMS la teneur en P.H pour une eau potable doit être située entre : 6,5 et 9. La conductivité quant à elle permet d'apprécier la quantité de sels dissouts dans l'eau présente dans les trois forages. Là encore, les valeurs sont largement en deçà du niveau guide recommandé par l'OMS.

Si l'eau très pure est un isolant qui oppose une grande résistance au passage de l'électricité, il n'en est plus de même lorsqu'elle est chargée en sels minéraux d'origine naturelle (calcium, magnésium, sodium, potassium). Ces indicateurs de conductibilité (Verhille 2013) bien que faible par rapport au niveau guide traduisent des traces de minéralisation et permettent de signaler de façon globale la présence probable d'une pollution. Le forage 3 est particulièrement concerné par cette situation. A titre de comparaison, l'analyse des échantillons d'eau prélevés dans le bassin des Iullemeden au Niger révèle, que les valeurs de la conductivité sont inférieures à $700 \mu \mathrm{S} . \mathrm{cm}-1$, donc 
faiblement minéralisées et par conséquent conformes aux normes OMS; (Saley Abdel K. et al. 2019).

L'analyse bactériologique a permis de mettre en évidence la pollution fécale de l'eau. Elle présente un bon moyen pour contrôler l'efficacité des mesures de protection ou de traitement entreprises par les habitants. La détection de l'indicateur $E$. coli est une preuve incontestable de l'occurrence d'une contamination fécale récente et indique la présence potentielle de pathogènes entériques. Sans être parfait, E. coli reste à ce jour le meilleur indicateur microbien d'usage courant pour signaler les risques sanitaires associés à la consommation de l'eau des réseaux de distribution.

D'après la répartition topographique des points de prélèvement, nous relevons que les concentrations d'Escherichia coli décroissent plus avec l'altitude, tandis que la concentration des entérocoques intestinaux varient indépendamment de la situation du point d'eau par rapport à la proximité des eaux de mangroves, mais davantage en fonction de la proximité des latrines par rapport au point d'eau. Les entérocoques intestinaux sont en effet des micro-organismes vivants dans les matières fécales et plus résistants que les coliformes. La persistance des entérocoques dans divers types d'eau peut être supérieure à celle des autres organismes indicateurs (Edberg et al. 2000, OMS 2004), notamment à cause de leur résistance notoire aux agents désinfectants, ce qui fait d'eux des indicateurs privilégiés pour évaluer l'efficacité du traitement de l'eau (OMS, 2004). Certes, la mise en évidence de ces germes indicateurs de pollution fécale n'est pas forcément la preuve de la présence de pathogènes, mais elle permet de la suspecter fortement. C'est pour en avoir le cœur net qu'une enquête sanitaire a été menée.

\section{III.3- Forte prévalence des maladies hydriques en fonction du mode d'accès et d'utilisation de l'eau potable}

Au sens général, les maladies hydriques sont liées à la qualité de l'eau et à l'accès à l'eau potable. Il peut s'agir d'eaux utilisées pour la boisson, la cuisson, la baignade, ou encore pour se laver ou pour laver des objets. Le risque de transmission le plus direct est lié à l'eau de boisson. Le passage du pathogène peut aussi se faire via des blessures ou par le passage d'un parasite au travers de la peau. Le CIR (Centre International de l'Eau et de l'Assainissement) propose en 1988 une classification suivant l'implication de l'eau dans les cas d'infection :

- les maladies transmises par l'eau.

- Les maladies liées à l'absence d'eau.

-Les maladies à agents infectieux vivants dans l'eau. 


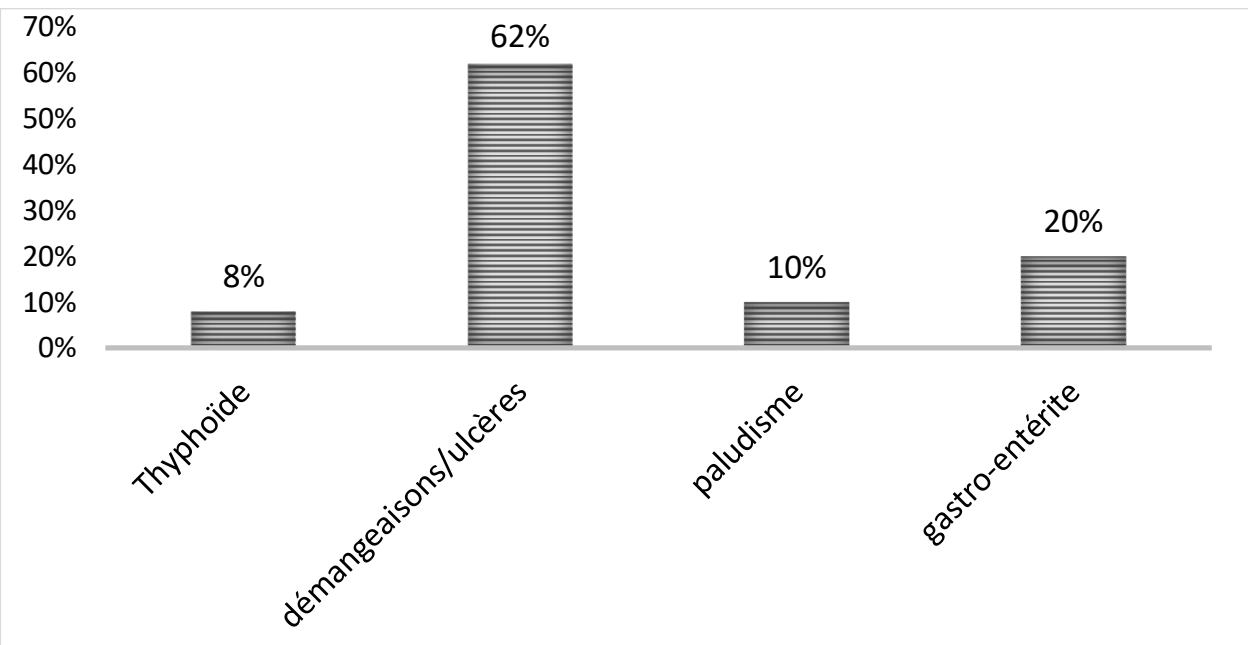

Figure 12: Répartition des maladies hydriques recensées auprès des ménages et dans les deux centres de santé de référence du quartier. Source: enquêtes de terrain, 2015

Nos enquêtes dans les centres de santé du quartier et dans les ménages ont permis de répertorier et de classifier les différentes maladies hydriques (fig.12)

$>$ Les maladies transmissibles par l'eau : Ces maladies sont causées par des agents infectieux provenant soit des aliments mal lavés, soit des hommes et des animaux à travers l'eau de boisson. Parmi ces maladies, les plus courantes observées à Nsong Ngogang sont : la fièvre typhoïde (8\%) et les gastro-entérites ou diarrhées $(20 \%)$.

$>$ Les maladies dues à la rareté de l'eau : Le manque ou l'absence d'un réseau d'assainissement crée des conditions favorables au développement de ces pathologies. Nous avons recensé dans ce groupe les maladies de la peau et les ulcères enregistrés sous l'expression "Came no go ». Ce sont les pathologies les plus courantes dans les ménages enquêtés $(62 \%)$.

$>$ Les maladies à vecteur liés à l'eau : Elles requièrent un agent infectant qui héberge l'agent infectieux pendant l'une des phases de son cycle vital. Il s'agit principalement des escargots et des crustacés, mais aussi des insectes (moustiques, mouches) vivant près des cours d'eaux. Parmi ces maladies, nous avons répertorié le paludisme (10\%).

L'enquête par questionnaire auprès les ménages montre que sur 90 ménages enquêtés, tous affirment que les démangeaisons (irruptions cutanées) sont causées par l'utilisation de l'eau de puits pour le bain. Ils attribuent également l'occurrence des gastro-entérites à la consommation de l'eau de forage. La figure 13 donne une répartition des maladies rencontrées en fonction de la source d'approvisionnement qui en est la cause probable essentiellement d'après les enquêtés. Les habitants semblent bien 
conscients des risques auxquels ils s'exposent, mais préfèrent y demeurer et se convaincre de l'efficacité de leurs mesures de protections.

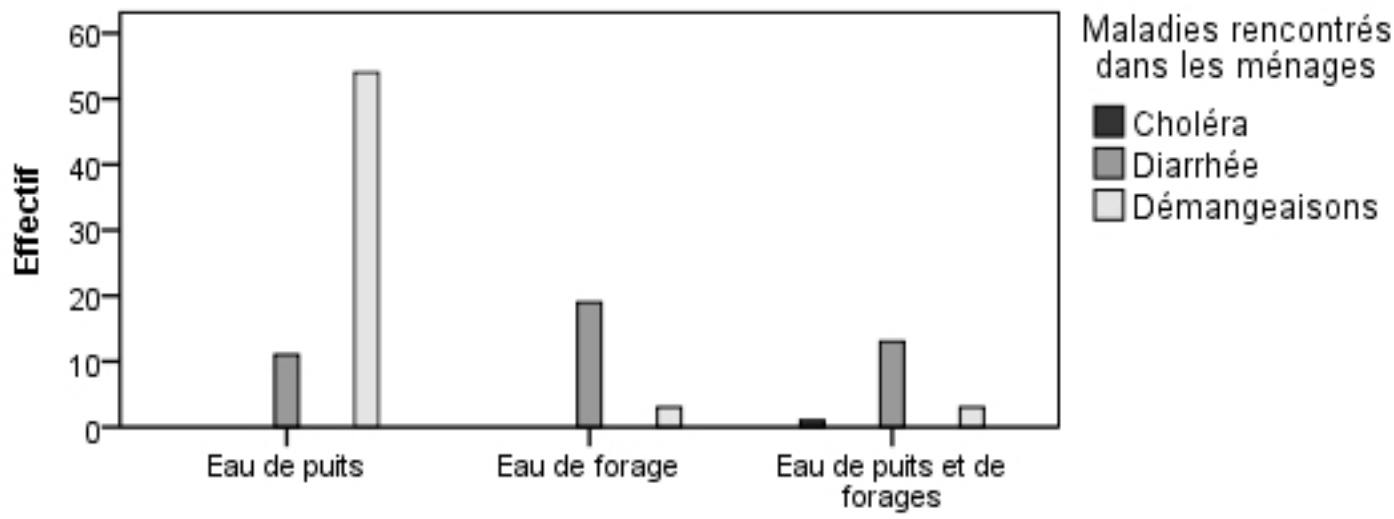

\section{Cause des maladies}

Figure 13: Répartition des maladies recensées en fonction de la source d'approvisionnement. Source: enquêtes de terrain, 2015

\section{IV - Discussion}

L'approvisionnement eau reste une gageure dans les quartiers précaires des villes du sud malgré les efforts des politiques internationales et nationales (Jaglin 2001, Kamgho Tezanou 2009, Nantchop Tenkap 2017). Les modèles marchands ont prouvé leurs limites et il est de plus en plus préconisé des modèles territorialisés ou en réseaux (Jaglin 2012). Au sud de Douala, la mise en réseau des puits ou des forages du quartier implique des solidarités constamment renouvelées à l'occasion de l'entretien du puits ou du traitement de l'eau. L'accès à l'eau est étroitement lié à l'accès à l'assainissement dans les quartiers précaires en milieu de plaine. Les eaux souillées par le mauvais drainage et les dépotoirs sauvages des déchets ménagers accentuent les vulnérabilités par rapport aux maladies hydriques (Biang Mbella 2006, Meva'a Abomo 2006). Le même constat a été fait dans les villes d'altitudes (Mpakam et al.2006). La prévalence des maladies hydriques est fonction de la vulnérabilité de chaque habitant par rapport à l'accessibilité (Yemmafouo et Ngouanet 2014). Les mêmes proportions ont été constatées entre autres à Ouagadougou (Dos Santos 2011, Agassounon et al. 2012), à Cotonou (Odoulami 2009), à Dschang-Cameroun (Yemmafouo et Ngouanet 2014), et dans le district de Ngabé au Congo (Essouli Olivier et al. 2019). Tout compte fait, les habitants des quartiers précaires développent de petites innovations face aux pénuries qu'il convient de soutenir par des approches territorialisées (Jaglin 2012, Baron et Tidjani Alou, 2011). 


\section{Conclusion}

L'objectif de ce travail était de mettre en évidence le déficit chronique en eau potable et ses implications sur la vie des habitants de mangroves. Il ressort des recherches menées que malgré les mesures prises pour apprivoiser l'eau en général, ces habitants n'ont pas réussi à assurer une qualité de l'eau potable capable de réduire le taux de prévalence des maladies hydriques dans leur milieu. Du point de vue naturel, le caractère inaccessible de l'espace à mangrove pour l'occupation humaine n'a pas suffi à dissuader leur installation. Bien au contraire, un accroissement exponentiel des surfaces bâties est observé dans cet espace amphibie en guise d'impact de l'étalement anarchique de la ville de Douala. La question d'accès aux services de base et notamment à l'eau potable s'est donc avérée pertinente. Exposés non seulement aux inondations mais aussi au manque d'eau potable, les habitants ont reproduit les pratiques d'approvisionnement en eau des quartiers précaires de Douala, mais avec une acuité plus marquée. L'eau des puits sommaire et des forages impropre à la consommation est en vedette. Les traitements appliqués à cette eau ne suffisent pas à dissiper les doutes. C'est donc sans surprise qu'une forte prévalence des maladies hydriques a été enregistrée ; les plus répandues étant les démangeaisons et irruptions cutanées $(60 \%)$ et les gastroentérites (20\%). Il reste à chercher à comprendre davantage cette résilience des populations de mangroves urbanisées.

\section{References:}

1. Agassounon M., Djikpo T., Ayi-Fanou L., Lozes E., Fadonougbo R., Anago G. D. J., Agbangla C. \& Ahanhanzo C. (2012). Impacts sanitaires liés à l'usage des eaux de puits, à l'assainissement et à l'aménagement à Gbôdjê dans l'arrondissement de Godomey au Bénin, International Journal of Biological and chemical sciences, 6(2), 592-602., available online at http://ajol.info/index.php/ijbcs.

2. Baron, C. \& Tidjani A. M. (2011). L'accès à l'eau en Afrique subsaharienne: au-delà des modèles, une pluralité d'innovations locales. Mondes en développement, 155(3), p.7-22. doi:10.3917/med.155.0007.

3. Biang Mbella. C., (2006). La problématique de l'approvisionnement en eau potable : essai d'analyse appliqué à la ville de Yabassi dans la province du Littoral. Mémoire présenté et soutenu en vue de l'obtention du diplôme de Maitrise en Géographie, Université de Dschang, 123p.

4. Dos Santos, S. (2011). Les risques sanitaires liés aux usages domestiques de l'eau. Représentations sociales mossi à Ouagadougou (Burkina Faso). Natures Sciences Sociétés, vol. 19(2), 103-112. 
https://www.cairn.info/revue-natures-sciences-societes-2011-2-page103.htm.

5. Dzalla Ngangue Guy C., (2012). La mangrove de l'estuaire du Wouri (douala - Cameroun) : enjeux de l'anthropisation d'un écosystème humide tropical et impacts environnementaux. Thèse de Doctorat en Géographie, Université de Douala, 446p.

6. Eba, A. E. L., Kouame, K. J., Deh, S., Balliet, R., Touré, M., Anoh, A. K., \& Jourda, J. P. R. (2016). Évaluation De La Vulnerabilite A La Pollution D'une Eau De Surface Destinee A L'adduction D'eau Potable D'une Metropole. Cas De La Lagune Aghein A Abidjan,(Sud De La Cote D'ivoire). European Scientific Journal, 21p.

7. Edberg, S. C. L., Rice, E. W., Karlin, R. J., \& Allen, M. J. (2000). Escherichia coli: the best biological drinking water indicator for public health protection. Journal of applied microbiology, 88(S1), 106S$116 \mathrm{~S}$.

8. Essouli O. F.., Matini L.., Miyouna T., Fouroux M. A., Boudzoumou F. \& Faye S., (2019) Prospection Hydrogéologique et Géophysique du Système Aquifère du Plateau de Mbé, District de Ngabé, Congo. European Scientific Journal, 32p.

9. Jaglin Sylvy, (2001). L'eau potable dans les villes en développement : les modèles marchands face à la pauvreté. Revue Tiers Monde, tome $42, \mathrm{n}^{\circ} 166$, p. $275-303$.

10. Jaglin Sylvy, (2012). Services en réseaux et villes africaines : l'universalité par d'autres voies ? L'espace Géographique, tome 41, vol.1, p51-67.

11. Kamgho Tezanou Bruno M., (2009). L'accès à l'eau potable et à l'assainissement au Cameroun : situation actuelle, contraintes, enjeux et défis pour l'atteinte de l'OMD 7». Cameroun, 13p.

12. Meva'a Abomo. D, (2006). De l'abondance des ressources en eau a la rareté de l'eau potable dans les villes littorales du sud; un indicateur pertinent de la crise managériale de ces espaces urbains : l'exemple de douala au Cameroun. Laboratoire de recherche en Géographie, Université de Douala, 17p.

13. Mpakam H. G., Kamgang K., Kouam K. G.-R., Tamo T. \& Ekodeck G. E., (2006). L'accès à l'eau potable et à l'assainissement dans les villes des pays en développement: cas de Basoussam (Cameroun), VertigO - la revue électronique en sciences de l'environnement, 7(2).

14. Nantchop Tenkap V. L. (2017). Politiques publiques de l'eau et gouvernance urbaine. Acteurs et enjeux de l'accès à l'eau potable des populations à Douala (Cameroun), thèse de Géographie, Université Paris 1 Panthéon-Sorbonne. 
15. Ngnikam, E., Mougoue, B., \& Tietche, F. (2007). Eau, Assainissement et impact sur la santé: étude de cas d'un écosystème urbain à Yaoundé. Actes des JSIRAUF, Hanoi, 6-9.

16. Odoulami Leocadie, (2009). La problématique de l'eau potable et la santé humaine dans la ville de Cotonou (République du Bénin). Thèse de Doctorat Unique pour l'obtention du grade de Docteur ès Lettres de l'Université d'Abomey-Calavi en Géographie et Gestion de l'Environnement, Université d'Abomey-Calavi, 230p.

17. OMS, (2004). Directives de qualité pour l'eau de boisson troisième édition volume 1 recommandations, Genève, $110 \mathrm{p}$.

18. Saley, A. K. H., Sandao, I., Michelot, J. L., \& Boureïma, O., (2019) Apport des Paramètres Physico-Chimiques des Eaux à l'amélioration de la Connaissance de l'aquifère du Continental Intercalaire/Continental Hamadien de la Région de Tahoua (Bassin des Iullemeden, Niger). European Scientific Journal, 25p.

19. Tegofack R, (2000). Problématique de l'approvisionnement en eau dans la ville de Yaoundé : cas de la région desservie par le réservoir d'Etoudi. Thèse de Masters of sciences en gestion de l'eau, Université de Dschang, 75p.

20. Temgoua Tiayo. H., (2006). Eau et environnement en milieu urbain : cas de Dschang à l'Ouest-Cameroun. Mémoire présenté en vue de l'obtention du diplôme d'étude approfondie, Université de Dschang, $86 \mathrm{p}$.

21. Verhille Sophie, (2013). Les indicateurs microbiens dans l'évaluation de l'eau potable : interpréter les résultats de laboratoire et comprendre leur signification pour la santé publique. Centre de collaboration nationale en santé environnementale, 13p.

22. Yemmafouo A., Ngouanet Ch., (2014). Géographicité et déterminants sociaux de la croissance des maladies hydriques dans la ville de Dschang (Ouest-Cameroun), in Nguendo Yongsi B., Mimche H. (dir), Urbanisation, environnement et enjeux sanitaires en Afrique. Editions Universitaires Européennes, Sarrebruck, 384p. 